\title{
The LsVe1L allele provides a molecular marker for resistance to Verticillium dahliae race 1 in lettuce
}

Patrik Inderbitzin ${ }^{1,2+}$, Marilena Christopoulou ${ }^{3+}$, Dean Lavelle ${ }^{3}$, Sebastian Reyes-Chin-Wo ${ }^{3}$, Richard W. Michelmore ${ }^{3,4}$, Krishna V. Subbarao ${ }^{1 *}$ and Ivan Simko ${ }^{5^{*}}$ (D)

\begin{abstract}
Background: Verticillium wilt caused by the fungus Verticillium dahliae race 1 is among the top disease concerns for lettuce in the Salinas and Pajaro Valleys of coastal central California. Resistance of lettuce against $V$. dahliae race 1 was previously mapped to the single dominant Verticillium resistance 1 (Vr 1 ) locus. Lines of tomato resistant to race 1 are known to contain the closely linked Vel and Ve2 genes that encode receptor-like proteins with extracellular leucine-rich repeats; the Ve1 and Ve2 proteins act antagonistically to provide resistance against $V$. dahliae race 1 . The Vrl locus in lettuce contains a cluster of several genes with sequence similarity to the tomato Ve genes. We used genome sequencing and/or PCR screening along with pathogenicity assays of 152 accessions of lettuce to investigate allelic diversity and its relationship to race 1 resistance in lettuce.

Results: This approach identified a total of four Ve genes: LsVe1, LsVe2, LsVe3, and LsVe4. The majority of accessions, however, contained a combination of only three of these LsVe genes clustered on chromosomal linkage group 9 (within $\sim 25 \mathrm{~kb}$ in the resistant cultivar La Brillante and within $\sim 127 \mathrm{~kb}$ in the susceptible cultivar Salinas).

Conclusions: A single allele, LsVe1L, was present in all resistant accessions and absent in all susceptible accessions. This allele can be used as a molecular marker for $V$. dahliae race 1 resistance in lettuce. A PCR assay for rapid detection of race 1 resistance in lettuce was designed based on nucleotide polymorphisms. Application of this assay allows identification of resistant genotypes in early stages of plant development or at seed-level without time- and laborintensive testing in the field.
\end{abstract}

Keywords: Lactuca sativa, Genomics, Marker-assisted selection, Plant breeding, Wilt resistance

\section{Background}

The Salinas and Pajaro Valleys of coastal central California are among the most important lettuce-producing regions in the United States [1]. One of the top disease concerns for lettuce in the area is Verticillium wilt caused by the fungus Verticillium dahliae [2, 3], which is a soilborne pathogen with a wide host range that also includes artichoke, cotton, eggplant, hops, potato, sunflower, tobacco, and tomato [4,

\footnotetext{
* Correspondence: kvsubbarao@ucdavis.edu; Ivan.Simko@ars.usda.gov ${ }^{\dagger}$ Patrik Inderbitzin and Marilena Christopoulou contributed equally to this work.

'Department of Plant Pathology, University of California, Davis, CA 95616, USA

${ }^{5}$ United States Department of Agriculture, Agricultural Research Service, Crop Improvement and Protection Research Unit, Salinas, CA 93905, USA
} Full list of author information is available at the end of the article
5]. Two races of $V$. dahliae occur in coastal central California based on their differential virulence on cultivar La Brillante [6]; however, race 1 is more prevalent and economically important than race 2 [7]. In tomato, race 1 of $V$. dahliae carries Ave1 that is recognized by $V e 1$ in resistant genotypes [8]. Ve genes encode receptor-like proteins (RLPs) with extracellular leucine-rich repeats $[9,10]$; such RLPs are widespread in land plants [11]. In addition to Ve1, tomato also contains the closely linked paralog $\mathrm{Ve} 2$; their encoded RLPs work antagonistically to confer resistance to $V$. dahliae race 1 [12]. Several Ve paralogs also confer resistance in otherwise $V$. dahliae-susceptible species including cotton [13], potato [14, 15], hops, and wild eggplant [11], but it is unknown whether they function analogously to the tomato $V e$ genes in conferring $V$. dahliae race 1 
resistance. In lettuce, resistance to $V$. dahliae race 1 was originally identified in the Batavia-type cultivar, La Brillante, as conferred by a single dominant locus (Verticillium resistance 1, Vr1) located on chromosomal linkage group 9 [16]. The lettuce $V r 1$ locus contains several genes with sequence similarity to the $\mathrm{Ve}$ genes of tomato; it is very likely that one or more of these LsVe homologs are functional resistance genes.

The goals of this study were to identify the lettuce $V e$ allele(s) that play a role in resistance to $V$. dahliae race 1 and to develop PCR-based assays for marker-assisted selection. For this purpose, we analyzed the genome sequences of cultivars La Brillante (resistant to $V$. dahliae race 1 ) and the previously published Salinas [17] (iceberg type, susceptible to $V$. dahliae race 1 ). Subsequently, we sequenced and/or used allele-specific PCR screens of 150 additional lettuce accessions to identify the allele(s) of the LsVe genes that are exclusively present in resistant phenotypes.

\section{Results}

\section{Phenotypic evaluation of resistance in field tests}

One hundred and fifty accessions from ten horticultural types and L. serriola were evaluated in four field experiments. Twenty accessions (13.3\%) showed no disease symptoms and were considered resistant. The proportion of disease incidence in susceptible accessions ranged from 0.07 to 1.00 , with a mean disease incidence of $0.43( \pm 0.02)$. There was a substantial difference in the distribution of resistant phenotypes across horticultural types. Among horticultural types with at least five tested accessions, the largest frequency of resistant accessions was found in Latin $(6 / 7=85.7 \%)$, followed by Batavia ( 2 / $6=33.3 \%)$, red leaf $(4 / 15=26.7 \%)$, and butterhead $(3 /$ $14=21.4 \%$; Table 1) types. In contrast, the lowest frequencies of resistant accessions were found in iceberg $(0 / 46=0 \%)$, romaine $(2 / 36=5.6 \%)$, and green leaf $(2 /$ $18=11.1 \%)$ types. Oil $(0 / 4=0 \%)$, stem $(1 / 3=33.3 \%)$ types, and L. serriola $(0 / 1=0 \%)$ had fewer than five tested accessions each. All oil type accessions were susceptible to the disease and had a very high disease incidence (0.98 in one accession, 1.00 in all others). Statistical analysis indicated that the frequency of resistant accessions was significantly $(p<0.01)$ higher than the overall frequency of $13.3 \%$ in Latin types, while it was significantly lower in iceberg types; however, the statistical power to detect significant differences for horticultural types with a small number of tested accessions is limited.

\section{Lettuce genome assemblies}

Genome assemblies were generated for 61 accessions of cultivated lettuce (Table 2). The assembly of cultivar La Brillante consisted of 41,939 scaffolds with a total length of $2.04 \mathrm{~Gb}$ and had an $\mathrm{L}_{50}$ of $90.84 \mathrm{~kb}$. The remaining 60 draft de novo assemblies consisted of 1.0 to $3.2 \mathrm{M}$ contigs (average $2.78 \mathrm{M}$ ) with a total length of 2.08 to $2.44 \mathrm{~Gb}$ (average $2.20 \mathrm{~Gb}$ ) and an $\mathrm{L}_{50}$ of 1.22 to $3.66 \mathrm{~kb}$ (average 1.6 $\mathrm{kb}$ ). Reads have been submitted to GenBank (BioProject PRJNA478460).

\section{Ve genes and alleles of cultivars La Brillante, Salinas, and 60 other accessions}

The expressed sequence tag marker QGD8I16.yg.ab1 at the Verticillium resistance $1(V r 1)$ locus in lettuce [15] was used to query the genome assemblies of the lettuce cultivars La Brillante and Salinas using BLASTn. Three hits $(\mathrm{e}=0.0)$ to scaffold linkage group 9 of the v8 reference assembly of the cultivar Salinas corresponded to three

Table 1 Difference among horticultural types in their phenotypic reaction to $V$. dahliae race 1 tested in field trials

\begin{tabular}{|c|c|c|c|c|c|c|c|c|}
\hline \multirow{2}{*}{$\begin{array}{l}\text { Horticultural type } \\
\text { or species }\end{array}$} & \multirow{2}{*}{$\begin{array}{l}\text { No. of tested } \\
\text { accessions }\end{array}$} & \multicolumn{5}{|c|}{ No. of accessions with disease incidence } & \multirow{2}{*}{$\begin{array}{l}\text { Frequency of } \\
\text { resistant accessions }\end{array}$} & \multirow{2}{*}{$\begin{array}{l}\text { Chi-square } \\
\text { test }^{\text {a }}\end{array}$} \\
\hline & & 0.00 (resistant) & $0.01-0.25$ & $0.26-0.50$ & $0.51-0.75$ & $0.76-1.00$ & & \\
\hline Batavia & 6 & 2 & & 4 & & & 0.33 & 1.6 \\
\hline Butterhead & 14 & 3 & 9 & 1 & & 1 & 0.21 & 0.8 \\
\hline Green leaf & 18 & 2 & 2 & 10 & 2 & 2 & 0.11 & 0.1 \\
\hline Iceberg & 46 & & 10 & 23 & 10 & 3 & 0.00 & $12.8^{*}$ \\
\hline Latin & 7 & 6 & 1 & & & & 0.86 & $19.0^{*}$ \\
\hline Oil & 4 & & & & & 4 & 0.00 & 1.1 \\
\hline Red leaf & 15 & 4 & & 3 & 4 & 4 & 0.27 & 2.0 \\
\hline Romaine & 36 & 2 & 9 & 20 & 4 & 1 & 0.06 & 2.2 \\
\hline Stem & 3 & 1 & & 2 & & & 0.33 & 0.8 \\
\hline Lactuca serriola & 1 & & & & 1 & & 0.00 & 0.3 \\
\hline Total & 150 & 20 & 31 & 63 & 21 & 15 & 0.13 & \\
\hline
\end{tabular}

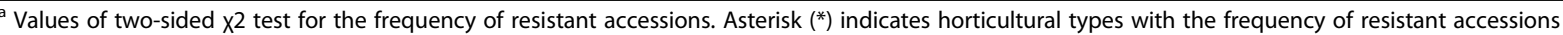
significantly different at experiment-wise $p<0.01$ from the overall frequency $(0.13)$ observed for all tested types. Note that the statistical test has a low to absent detection power for significant results for horticultural types with a very few tested accessions 
Table 2 Accessions used in this study sorted by horticultural type and accession name

\begin{tabular}{|c|c|c|c|c|c|c|c|c|c|c|c|c|}
\hline Type & Accession & Identifier & Genome & $\begin{array}{l}\text { Race } 1 \\
\text { resistance }\end{array}$ & LsVe1L & LsVe3L & LsVe4L & LsVerS & LsVe2S & LsVe3S & $\begin{array}{l}\text { No. of } \\
\text { tested plants }\end{array}$ & $\begin{array}{l}\text { Proportion of } \\
\text { symptomatic plants }\end{array}$ \\
\hline Batavia & Anuenue $^{\mathrm{a}, \mathrm{b}}$ & L2 & + & - & - & - & - & - & + & + & 90 & $0.26(0.18-0.35)$ \\
\hline Batavia & $\begin{array}{l}\text { Batavia Reine } \\
\text { des Glaces }{ }^{c, b}\end{array}$ & L90 & - & - & - & $N D^{i}$ & - & ND & ND & ND & 90 & $0.44(0.35-0.55)$ \\
\hline Batavia & Iceberg ${ }^{c, b}$ & L115 & - & - & - & ND & - & ND & ND & ND & 30 & $0.50(0.33-0.67)$ \\
\hline Batavia & La Brillante ${ }^{d, e}$ & $\begin{array}{l}10 G 364- \\
1\end{array}$ & + & + & + & + & + & - & - & - & $N T^{\mathrm{j}}$ & NT \\
\hline Batavia & La Brillante ${ }^{c, b}$ & L119 & - & + & + & ND & + & ND & ND & ND & 180 & $0.00(0.00-0.02)$ \\
\hline Batavia & Laurad, b & L43 & + & + & + & + & + & - & - & - & 60 & $0.00(0.00-0.06)$ \\
\hline Batavia & $\begin{array}{l}\text { Reines des } \\
\text { Glaces }{ }^{d, b} b\end{array}$ & L53 & + & - & - & - & - & + & + & + & 30 & $0.40(0.25-0.58)$ \\
\hline Butterhead & Allegiance $^{a, b}$ & L85 & - & - & - & ND & + & ND & ND & ND & 30 & $0.17(0.07-0.34)$ \\
\hline Butterhead & Ancora $^{a, f}$ & L240 & - & + & + & ND & + & ND & ND & ND & 60 & $0.00(0.00-0.06)$ \\
\hline Butterhead & Bennett ${ }^{\mathrm{a}, \mathrm{b}}$ & L93 & - & - & - & ND & + & ND & ND & ND & 30 & $0.20(0.10-0.37)$ \\
\hline Butterhead & $\mathrm{Bibb}^{\mathrm{d}, \mathrm{b}}$ & L26 & + & - & - & - & - & + & + & + & 30 & $0.07(0.02-0.21)$ \\
\hline Butterhead & Cobham Green ${ }^{\mathrm{a}, \mathrm{b}}$ & L6 & + & - & - & + & + & + & + & - & 30 & $0.80(0.63-0.91)$ \\
\hline Butterhead & $\begin{array}{l}\text { Dark Green } \\
\text { Boston- } C^{d, ~ b}\end{array}$ & L71 & + & - & - & - & + & + & + & - & 26 & $0.23(0.11-0.42)$ \\
\hline Butterhead & Grappa a, b & L106 & - & - & - & ND & - & ND & ND & ND & 30 & $0.07(0.02-0.21)$ \\
\hline Butterhead & Little Gem-G ${ }^{\text {d, b }}$ & L77 & + & + & + & + & + & - & - & - & 60 & $0.00(0.00-0.06)$ \\
\hline Butterhead & Margarita $^{\mathrm{d}, \mathrm{b}}$ & $\llcorner 45$ & + & - & - & - & + & + & + & - & 30 & $0.30(0.17-0.48)$ \\
\hline Butterhead & Mariska ${ }^{d, b}$ & $\llcorner 46$ & + & - & - & - & + & + & + & - & 29 & $0.24(0.12-0.42)$ \\
\hline Butterhead & Martin ${ }^{a, b}$ & L124 & - & - & - & ND & - & ND & ND & ND & 30 & $0.17(0.07-0.34)$ \\
\hline Butterhead & Ostinata $a^{a, b}$ & L127 & - & - & - & ND & + & ND & ND & ND & 30 & $0.07(0.02-0.21)$ \\
\hline Butterhead & Tania $a^{a, b}$ & L149 & - & - & - & ND & + & ND & ND & ND & 30 & $0.07(0.02-0.21)$ \\
\hline Butterhead & Tinto ${ }^{a, b}$ & L23 & + & + & + & + & + & - & - & - & 59 & $0.00(0.00-0.06)$ \\
\hline Green leaf & Alpine $e^{a, b}$ & L86 & - & - & - & ND & - & ND & ND & ND & 30 & $0.40(0.25-0.58)$ \\
\hline Green leaf & Franklin ${ }^{a, b}$ & L100 & - & - & - & ND & - & ND & ND & ND & 60 & $0.20(0.12-0.32)$ \\
\hline Green leaf & $\begin{array}{l}\text { Genecorps } \\
\text { Green, b }\end{array}$ & L103 & - & - & - & ND & - & ND & ND & ND & 30 & $0.60(0.42-0.75)$ \\
\hline Green leaf & Grand Rapids ${ }^{d, b}$ & L37 & + & - & - & - & - & + & + & + & 60 & $0.45(0.33-0.58)$ \\
\hline Green leaf & Green Vision, ${ }^{a}$ b & L109 & - & - & - & ND & - & ND & ND & ND & 30 & $0.20(0.10-0.37)$ \\
\hline Green leaf & Haciendad, b & $\llcorner 40$ & + & - & - & - & - & + & + & + & 30 & $0.97(0.83-0.99)$ \\
\hline Green leaf & Plymouth $^{\mathrm{a}, \mathrm{b}}$ & L19 & + & + & + & + & + & - & - & - & 120 & $0.00(0.00-0.03)$ \\
\hline Green leaf & Pybas Green ${ }^{a, b}$ & L133 & - & - & - & ND & - & ND & ND & ND & 30 & $0.37(0.22-0.54)$ \\
\hline Green leaf & Salad Bowl $\left.\right|^{a, f}$ & L307 & - & - & - & ND & - & ND & ND & ND & 27 & $0.41(0.25-0.59)$ \\
\hline Green leaf & Shining Star ${ }^{\mathrm{a}, \mathrm{b}}$ & L141 & - & - & - & ND & - & ND & ND & ND & 30 & $0.50(0.33-0.67)$ \\
\hline Green leaf & Slobolt ${ }^{d, b}$ & L56 & + & - & - & - & - & + & + & + & 29 & $0.45(0.28-0.62)$ \\
\hline Green leaf & Tehama ${ }^{a, b}$ & L150 & - & - & - & ND & - & ND & ND & ND & 30 & $0.47(0.30-0.64)$ \\
\hline Green leaf & Tropicana $^{\mathrm{d}, \mathrm{b}}$ & L58 & + & - & - & - & - & + & + & + & 30 & $0.70(0.52-0.83)$ \\
\hline Green leaf & Two Star ${ }^{\mathrm{a}, \mathrm{b}}$ & L25 & + & - & - & - & - & + & + & + & 150 & $0.39(0.31-0.47)$ \\
\hline Green leaf & $\begin{array}{l}\text { Waldmann's } \\
\text { Green a, b }\end{array}$ & L160 & - & - & - & ND & - & ND & ND & ND & 30 & $0.50(0.33-0.67)$ \\
\hline Green leaf & $\begin{array}{l}\text { Waldmans } \\
\text { Green- } G^{d, b}\end{array}$ & L84 & + & - & - & - & - & + & + & + & 30 & $0.37(0.22-0.54)$ \\
\hline Green leaf & $\begin{array}{l}\text { Western } \\
\text { Green }^{\mathrm{a}, \mathrm{b}}\end{array}$ & L161 & - & - & - & ND & - & ND & ND & ND & 30 & $0.90(0.74-0.97)$ \\
\hline
\end{tabular}


Table 2 Accessions used in this study sorted by horticultural type and accession name (Continued)

\begin{tabular}{|c|c|c|c|c|c|c|c|c|c|c|c|c|}
\hline Type & Accession & Identifier & Genome & $\begin{array}{l}\text { Race } 1 \\
\text { resistance }\end{array}$ & LsVe1L & LsVe3L & LsVe4L & LsVe1S & LsVe2S & LsVe3S & $\begin{array}{l}\text { No. of } \\
\text { tested plants }\end{array}$ & $\begin{array}{l}\text { Proportion of } \\
\text { symptomatic plants }\end{array}$ \\
\hline Green leaf & Xena ${ }^{d, b}$ & L63 & + & + & + & + & + & - & - & - & 59 & $0.00(0.00-0.06)$ \\
\hline Iceberg & Autumn Gold ${ }^{d, b}$ & L66 & + & - & - & - & - & + & + & + & 28 & $0.54(0.36-0.70)$ \\
\hline Iceberg & Bayview ${ }^{a, b}$ & L92 & - & - & - & ND & - & ND & ND & ND & 30 & $0.30(0.17-0.48)$ \\
\hline Iceberg & Big Ben ${ }^{a, b}$ & L94 & - & - & - & ND & - & ND & ND & ND & 30 & $0.27(0.14-0.44)$ \\
\hline Iceberg & Calicel $^{\mathrm{d}, \mathrm{b}}$ & L27 & + & - & - & - & - & + & + & + & 26 & $0.54(0.35-0.71)$ \\
\hline Iceberg & Calmar ${ }^{a, b}$ & L5 & + & - & - & - & - & + & + & + & 30 & $0.50(0.33-0.67)$ \\
\hline Iceberg & Cannery Row $w^{a, b}$ & L163 & - & - & - & ND & - & ND & ND & ND & 30 & $0.20(0.10-0.37)$ \\
\hline Iceberg & Cisco $^{d, b}$ & L30 & + & - & - & - & - & + & + & + & 26 & $0.65(0.46-0.81)$ \\
\hline Iceberg & Coolguard ${ }^{d, b}$ & L31 & + & - & - & - & - & + & + & + & 28 & $0.86(0.69-0.94)$ \\
\hline Iceberg & Corona $^{a, b}$ & L169 & - & - & - & ND & - & ND & ND & ND & 30 & $0.20(0.10-0.37)$ \\
\hline Iceberg & Diplomat $^{d, b}$ & L34 & + & - & - & - & - & + & + & + & 29 & $0.55(0.38-0.72)$ \\
\hline Iceberg & Durango $\mathrm{o}^{\mathrm{a}, \mathrm{b}}$ & L173 & - & - & - & ND & - & ND & ND & ND & 30 & $0.30(0.17-0.48)$ \\
\hline Iceberg & Early Bird ${ }^{a, b}$ & L35 & + & - & - & - & - & + & + & + & 30 & $0.40(0.25-0.58)$ \\
\hline Iceberg & El Dorado $a, b$ & L174 & - & - & - & ND & - & ND & ND & ND & 30 & $0.20(0.10-0.37)$ \\
\hline Iceberg & Empire $^{a, b}$ & L175 & - & - & - & ND & - & ND & ND & ND & 30 & $0.30(0.17-0.48)$ \\
\hline Iceberg & Grand Slam ${ }^{a, b}$ & L105 & - & - & - & ND & - & ND & ND & ND & 30 & $0.27(0.14-0.44)$ \\
\hline Iceberg & Great Lakes ${ }^{\mathrm{a}, \mathrm{b}}$ & L107 & - & - & - & ND & - & ND & ND & ND & 30 & $0.40(0.25-0.58)$ \\
\hline Iceberg & $\begin{array}{l}\text { Great Lakes 659- } \\
G^{d, b}\end{array}$ & L73 & + & - & - & - & - & + & + & + & 30 & $0.37(0.22-0.54)$ \\
\hline Iceberg & Hallmark ${ }^{\mathrm{a}, \mathrm{b}}$ & L111 & - & - & - & ND & - & ND & ND & ND & 30 & $0.30(0.17-0.48)$ \\
\hline Iceberg & Home Run ${ }^{a, b}$ & L182 & - & - & - & ND & - & ND & ND & ND & 30 & $0.37(0.22-0.54)$ \\
\hline Iceberg & $\operatorname{Icon}^{\mathrm{a}, \mathrm{b}}$ & L116 & - & - & - & ND & - & ND & ND & ND & 30 & $0.30(0.17-0.48)$ \\
\hline Iceberg & Ithaca ZAA-C $C^{d, b}$ & L74 & + & - & - & - & - & + & + & + & 30 & $0.83(0.66-0.93)$ \\
\hline Iceberg & Laguna Fresca ${ }^{a, b}$ & L120 & - & - & - & ND & - & ND & ND & ND & 30 & $0.17(0.07-0.34)$ \\
\hline Iceberg & Legend $^{\mathrm{a}, \mathrm{b}}$ & L121 & - & - & - & ND & - & ND & ND & ND & 30 & $0.30(0.17-0.48)$ \\
\hline Iceberg & Liberty $^{a, b}$ & L122 & - & - & - & ND & - & ND & ND & ND & 30 & $0.37(0.22-0.54)$ \\
\hline Iceberg & Mesa $659-C^{d, b}$ & L78 & + & - & - & - & - & + & + & + & 30 & $0.80(0.63-0.91)$ \\
\hline Iceberg & Monument ${ }^{\mathrm{a}, \mathrm{b}}$ & L125 & - & - & - & ND & - & ND & ND & ND & 30 & $0.17(0.07-0.34)$ \\
\hline Iceberg & Pacific ${ }^{a, b}$ & L128 & - & - & - & ND & - & ND & ND & ND & 60 & $0.18(0.11-0.30)$ \\
\hline Iceberg & Primus $^{a, b}$ & L20 & + & - & - & - & - & + & + & + & 30 & $0.57(0.39-0.73)$ \\
\hline Iceberg & Salinas $^{\mathrm{g}}$ & $N A^{h}$ & NA & - & - & - & - & + & + & + & NT & NT \\
\hline Iceberg & Salinas ${ }^{c, b}$ & L138 & - & - & - & ND & - & ND & ND & ND & 180 & $0.55(0.48-0.62)$ \\
\hline Iceberg & Salinas $88^{c, b}$ & L139 & - & - & - & ND & - & ND & ND & ND & 120 & $0.48(0.40-0.57)$ \\
\hline Iceberg & Salinas $88-\mathrm{G}^{\mathrm{d}, \mathrm{b}}$ & L81 & + & - & - & - & - & + & + & + & 30 & $0.40(0.25-0.58)$ \\
\hline Iceberg & Sharp Shooter ${ }^{a, b}$ & L140 & - & - & - & ND & - & ND & ND & ND & 30 & $0.60(0.42-0.75)$ \\
\hline Iceberg & Silverado a, b & L143 & - & - & - & ND & - & ND & ND & ND & 30 & $0.30(0.17-0.48)$ \\
\hline Iceberg & Sniper ${ }^{a, b}$ & L144 & - & - & - & ND & - & ND & ND & ND & 60 & $0.55(0.42-0.67)$ \\
\hline Iceberg & Sun Devil ${ }^{a, b}$ & L146 & - & - & - & ND & - & ND & ND & ND & 30 & $0.20(0.10-0.37)$ \\
\hline Iceberg & Sure Shot ${ }^{a, b}$ & L148 & - & - & - & ND & - & ND & ND & ND & 30 & $0.20(0.10-0.37)$ \\
\hline Iceberg & Telluride $^{\mathrm{a}, \mathrm{b}}$ & L151 & - & - & - & ND & - & ND & ND & ND & 90 & $0.34(0.25-0.45)$ \\
\hline Iceberg & Tiber $^{a, b}$ & L152 & - & - & - & ND & - & ND & ND & ND & 60 & $0.53(0.41-0.65)$ \\
\hline Iceberg & Tribute $^{a, b}$ & L153 & - & - & - & ND & - & ND & ND & ND & 30 & $0.40(0.25-0.58)$ \\
\hline Iceberg & Trojan ${ }^{a, b}$ & L155 & - & - & - & ND & - & ND & ND & ND & 30 & $0.30(0.17-0.48)$ \\
\hline Iceberg & Vandenberg ${ }^{a, b}$ & L156 & - & - & - & ND & - & ND & ND & ND & 30 & $0.47(0.30-0.64)$ \\
\hline
\end{tabular}


Table 2 Accessions used in this study sorted by horticultural type and accession name (Continued)

\begin{tabular}{|c|c|c|c|c|c|c|c|c|c|c|c|c|}
\hline Type & Accession & Identifier & Genome & $\begin{array}{l}\text { Race } 1 \\
\text { resistance }\end{array}$ & LsVe1L & LsVe3L & LsVe4L & LsVe1S & LsVe2S & LsVe3S & $\begin{array}{l}\text { No. of } \\
\text { tested plants }\end{array}$ & $\begin{array}{l}\text { Proportion of } \\
\text { symptomatic plants }\end{array}$ \\
\hline Iceberg & Vanguard $^{a, b}$ & L157 & - & - & - & ND & - & ND & ND & ND & 30 & $0.20(0.10-0.37)$ \\
\hline Iceberg & Vanguard- $C^{d, b}$ & L82 & + & - & - & - & - & + & + & + & 30 & $0.30(0.17-0.48)$ \\
\hline Iceberg & Vanguard- $G^{\mathrm{d}, \mathrm{b}}$ & L83 & + & - & - & - & - & + & + & + & 30 & $0.23(0.12-0.41)$ \\
\hline Iceberg & Venus $^{\mathrm{a}, \mathrm{b}}$ & L183 & - & - & - & ND & - & ND & ND & ND & 30 & $0.47(0.30-0.64)$ \\
\hline Iceberg & Winterhaven $^{\mathrm{d}, \mathrm{b}}$ & L61 & + & - & - & - & - & + & + & + & 23 & $0.61(0.41-0.78)$ \\
\hline Latin & $\begin{array}{l}\text { Barnwood Gem }{ }^{a} \\
b\end{array}$ & L89 & - & + & + & ND & + & ND & ND & ND & 60 & $0.00(0.00-0.06)$ \\
\hline Latin & Brigade $^{a, b}$ & L97 & - & - & - & ND & - & ND & ND & ND & 30 & $0.17(0.07-0.34)$ \\
\hline Latin & Gallega $a^{a, b}$ & L102 & - & + & + & ND & + & ND & ND & ND & 60 & $0.00(0.00-0.06)$ \\
\hline Latin & Eruption c, b & L9 & + & + & + & + & + & - & - & - & 120 & $0.00(0.00-0.03)$ \\
\hline Latin & Little Gem, b & L123 & - & + & + & ND & + & ND & ND & ND & 60 & $0.00(0.00-0.06)$ \\
\hline Latin & Little Gem ${ }^{\mathrm{d}, \mathrm{b}}$ & $\llcorner 44$ & + & + & + & + & + & - & - & - & 60 & $0.00(0.00-0.06)$ \\
\hline Latin & Pavane $e^{a, b}$ & L16 & + & + & + & + & + & - & - & - & 90 & $0.00(0.00-0.04)$ \\
\hline Oil & PI $250020^{a, b}$ & L17 & + & - & - & - & - & + & + & + & 30 & $1.00(0.89-1.00)$ \\
\hline Oil & PI $251245^{\mathrm{d}, \mathrm{b}}$ & L50 & + & - & - & - & - & + & + & + & 30 & $1.00(0.89-1.00)$ \\
\hline Oil & PI $251245^{a, b}$ & L18 & - & - & - & ND & - & ND & ND & ND & 30 & $1.00(0.89-1.00)$ \\
\hline Oil & PI $251246^{c, b}$ & L131 & - & - & - & ND & - & ND & ND & ND & 60 & $0.98(0.91-1.00)$ \\
\hline Red leaf & Aragon Red ${ }^{a, b}$ & L87 & - & - & - & ND & - & ND & ND & ND & 30 & $0.47(0.30-0.64)$ \\
\hline Red leaf & Battalion ${ }^{a, b}$ & L91 & - & + & + & ND & + & ND & ND & ND & 60 & $0.00(0.00-0.06)$ \\
\hline Red leaf & Big Red ${ }^{a, b}$ & L95 & - & - & - & ND & - & ND & ND & ND & 30 & $1.00(0.89-1.00)$ \\
\hline Red leaf & Deep Red ${ }^{d, b}$ & L32 & + & - & - & - & - & + & + & + & 30 & $0.80(0.63-0.91)$ \\
\hline Red leaf & Lolla Rossa $a^{a, b}$ & L14 & + & + & + & + & + & - & - & - & 60 & $0.00(0.00-0.06)$ \\
\hline Red leaf & Merlot $^{a, b}$ & L15 & + & + & + & + & + & - & - & - & 90 & $0.00(0.00-0.04)$ \\
\hline Red leaf & New $\operatorname{Red}^{\mathrm{a}, \mathrm{b}}$ & L126 & - & - & - & ND & - & ND & ND & ND & 30 & $0.97(0.83-0.99)$ \\
\hline Red leaf & Prizehead ${ }^{a, b}$ & L132 & - & - & - & ND & - & ND & ND & ND & 30 & $0.70(0.52-0.83)$ \\
\hline Red leaf & Red Fox ${ }^{d, b}$ & L51 & + & - & - & - & - & + & + & + & 30 & $0.70(0.52-0.83)$ \\
\hline Red leaf & Red Grenoble $\mathrm{a}^{\mathrm{a}, \mathrm{b}}$ & L134 & - & - & - & ND & + & ND & ND & ND & 60 & $0.30(0.20-0.43)$ \\
\hline Red leaf & Red Rage $^{a, b}$ & L135 & - & - & - & ND & - & ND & ND & ND & 30 & $0.97(0.83-0.99)$ \\
\hline Red leaf & Red Tide ${ }^{a, b}$ & L136 & - & - & - & ND & - & ND & ND & ND & 30 & $0.57(0.39-0.73)$ \\
\hline Red leaf & Red Tide ${ }^{d, b}$ & L52 & + & - & - & - & - & + & + & + & 30 & $0.43(0.27-0.61)$ \\
\hline Red leaf & Sentry ${ }^{a, b}$ & L21 & + & + & + & + & + & - & - & - & 180 & $0.00(0.00-0.02)$ \\
\hline Red leaf & $\begin{array}{l}\text { Western Red } \\
\text { Leaf }{ }^{d, b}\end{array}$ & L60 & + & - & - & - & - & + & + & + & 30 & $0.53(0.36-0.70)$ \\
\hline Romaine & Annapolis, b & L1 & + & + & + & + & + & - & - & - & 90 & $0.00(0.00-0.04)$ \\
\hline Romaine & Avalanche $e^{c, b}$ & L88 & - & - & - & ND & - & ND & ND & ND & 90 & $0.22(0.15-0.32)$ \\
\hline Romaine & $\begin{array}{l}\text { Blonde Lente } \\
\text { a Monter }{ }^{c, b}\end{array}$ & $\llcorner 4$ & + & - & - & - & - & + & + & + & 30 & $0.70(0.52-0.83)$ \\
\hline Romaine & Brave Heart ${ }^{c, b}$ & L96 & - & - & - & ND & - & ND & ND & ND & 60 & $0.32(0.21-0.44)$ \\
\hline Romaine & Caesar $^{c, b}$ & L98 & - & - & - & ND & - & ND & ND & ND & 30 & $0.17(0.07-0.34)$ \\
\hline Romaine & Camino Real ${ }^{c, b}$ & L162 & - & - & - & ND & - & ND & ND & ND & 30 & $0.37(0.22-0.54)$ \\
\hline Romaine & Clemente ${ }^{c, b}$ & L166 & - & - & - & ND & - & ND & ND & ND & 30 & $0.20(0.10-0.37)$ \\
\hline Romaine & Coastal Star ${ }^{c, b}$ & L167 & - & - & - & ND & - & ND & ND & ND & 30 & $0.50(0.33-0.67)$ \\
\hline Romaine & Conquistador, & L168 & - & - & - & ND & - & ND & ND & ND & 30 & $0.27(0.14-0.44)$ \\
\hline Romaine & Costa Rica \# $4^{\mathrm{d}, \mathrm{b}}$ & L70 & + & - & - & - & - & + & + & + & 30 & $0.43(0.27-0.61)$ \\
\hline
\end{tabular}


Table 2 Accessions used in this study sorted by horticultural type and accession name (Continued)

\begin{tabular}{|c|c|c|c|c|c|c|c|c|c|c|c|c|}
\hline Type & Accession & Identifier & Genome & $\begin{array}{l}\text { Race } 1 \\
\text { resistance }\end{array}$ & LsVerl & LsVe3L & LsVe4L & LsVels & LsVe2S & LsVe3S & $\begin{array}{l}\text { No. of } \\
\text { tested plants }\end{array}$ & $\begin{array}{l}\text { Proportion of } \\
\text { symptomatic plants }\end{array}$ \\
\hline Romaine & Costa Rica \#4, b & L170 & - & - & - & ND & - & ND & ND & ND & 30 & $0.33(0.19-0.51)$ \\
\hline Romaine & Darkland $\mathrm{EL}^{\mathrm{c}, \mathrm{b}}$ & L171 & - & - & - & ND & - & ND & ND & ND & 30 & $0.37(0.22-0.54)$ \\
\hline Romaine & Defender, b & L8 & + & + & + & + & + & - & - & - & 90 & $0.00(0.00-0.04)$ \\
\hline Romaine & EXP1752a, b & L99 & - & - & - & ND & - & ND & ND & ND & 30 & $0.17(0.07-0.34)$ \\
\hline Romaine & Flashy Troutback, & L10 & + & - & - & + & + & - & + & - & 30 & $0.30(0.17-0.48)$ \\
\hline Romaine & Fresh Heart ${ }^{c, b}$ & L101 & - & - & - & ND & - & ND & ND & ND & 30 & $0.37(0.22-0.54)$ \\
\hline Romaine & Green Forest ${ }^{c, f}$ & L189 & - & - & - & ND & - & ND & ND & ND & 28 & $0.46(0.30-0.64)$ \\
\hline Romaine & Green Towers ${ }^{\mathrm{c}, \mathrm{b}}$ & L108 & - & - & - & ND & - & ND & ND & ND & 60 & $0.20(0.10-0.37)$ \\
\hline Romaine & Green Towers ${ }^{\mathrm{d}, \mathrm{b}}$ & L39 & + & - & - & - & - & + & + & + & 30 & $0.23(0.12-0.41)$ \\
\hline Romaine & Hearts Delightc, b & L112 & - & - & - & ND & - & ND & ND & ND & 150 & $0.31(0.24-0.38)$ \\
\hline Romaine & Heavy Heart ${ }^{c, b}$ & L113 & - & - & - & ND & - & ND & ND & ND & 30 & $0.30(0.17-0.48)$ \\
\hline Romaine & King Henry ${ }^{c, b}$ & L117 & - & - & - & ND & - & ND & ND & ND & 30 & $0.20(0.10-0.37)$ \\
\hline Romaine & $\underset{b}{\text { King Louie } 2005^{d}}$ & L76 & + & - & - & - & - & + & + & + & 30 & $0.83(0.66-0.93)$ \\
\hline Romaine & Klamath, $\mathrm{a}$ & L118 & - & - & - & ND & - & ND & ND & ND & 30 & $0.17(0.07-0.34)$ \\
\hline Romaine & Lee $T a l^{c, b}$ & L12 & + & - & - & - & + & + & + & - & 59 & $0.17(0.09-0.28)$ \\
\hline Romaine & Lobjoits $\operatorname{Cos}^{c, b}$ & L13 & + & - & - & - & - & + & + & + & 90 & $0.53(0.43-0.63)$ \\
\hline Romaine & 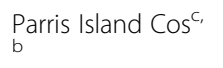 & L129 & - & - & - & ND & - & ND & ND & ND & 60 & $0.45(0.33-0.58)$ \\
\hline Romaine & $\begin{array}{l}\text { Parris Island Cos } \\
714 \text { (PIC714), }\end{array}$ & L130 & - & - & - & ND & - & ND & ND & ND & 30 & $0.40(0.25-0.58)$ \\
\hline Romaine & $\begin{array}{l}\text { Parris Island } \\
\text { Cos- } \mathrm{G}^{\mathrm{d}, \mathrm{b}}\end{array}$ & L80 & - & - & - & ND & - & ND & ND & ND & 30 & $0.43(0.27-0.61)$ \\
\hline Romaine & Passport ${ }^{d, b}$ & L64 & + & - & - & - & - & + & + & + & 30 & $0.50(0.33-0.67)$ \\
\hline Romaine & PI $171674^{d, b}$ & L49 & + & - & - & - & - & + & + & + & 90 & $0.32(0.23-0.42)$ \\
\hline Romaine & SM09A $A^{c, b}$ & L22 & + & - & - & - & - & + & + & + & 30 & $0.60(0.42-0.75)$ \\
\hline Romaine & Sunbelt ${ }^{c, b}$ & L184 & - & - & - & ND & - & ND & ND & ND & 30 & $0.40(0.25-0.58)$ \\
\hline Romaine & Triple Threat ${ }^{c, b}$ & L24 & + & - & - & - & - & + & + & + & 60 & $0.58(0.46-0.70)$ \\
\hline Romaine & Triton ${ }^{c, b}$ & L154 & - & - & - & ND & - & ND & ND & ND & 30 & $0.47(0.30-0.64)$ \\
\hline Romaine & $\mathrm{VJO}^{\mathrm{a}} \mathrm{R}^{\mathrm{a}} \mathrm{b}$ & L159 & - & - & - & ND & - & ND & ND & ND & 30 & $0.27(0.14-0.44)$ \\
\hline Stem & Balady Banha, b & L3 & + & + & + & + & + & - & - & - & 88 & $0.00(0.00-0.04)$ \\
\hline Stem & Celtuce $^{a, b}$ & L165 & - & - & - & ND & - & ND & ND & ND & 30 & $0.40(0.25-0.58)$ \\
\hline Stem & Celtuce- $G^{d, b}$ & L69 & + & - & - & - & - & + & + & + & 30 & $0.43(0.27-0.61)$ \\
\hline $\begin{array}{l}\text { Lactuca } \\
\text { serriola }\end{array}$ & $11-G 99^{c, f}$ & L185 & - & - & - & ND & + & ND & ND & ND & 30 & $0.70(0.52-0.83)$ \\
\hline
\end{tabular}

${ }^{\mathrm{a}}$ Germplasm Collection of USDA-ARS Salinas, CA

beeds

'Genome Wide Association Mapping Collection (GBS) of USDA-ARS Salinas, CA

dUC Davis Collection

eDark-grown seedlings

fLeaf tissue from field

${ }^{9}$ Reyes-Chin-Wo et al., 2017

${ }^{\mathrm{h}}$ Not applicable (NA), because this genome was sequenced previously (Reyes-Chin-Wo et al., 2017)

'Not determined (ND)

${ }^{\mathrm{j}}$ Not field tested in the present study (NT). Cultivars La Brillante and Salinas we previously confirmed to be resistant and susceptible, respectively, to $V$. dahliae race 1 (Hayes at al., 2011)

${ }^{\mathrm{k}} 95 \%$ confidence interval for the proportion of symptomatic plants is shown in parentheses 
open reading frames (ORFs) that were named LsVe1S (because it had the highest sequence similarity of the three paralogs to Ve1 of tomato), LsVe2S, and LsVe3S (Fig. 1). The encoded proteins were comprised of 1133 aa, 1041 aa, and 1039 aa for LsVe1S, LsVe2S, and LsVe3S, respectively, and including a signal peptide, 37 extracellular leucine-rich repeats, a transmembrane domain, and a cytoplasmic region inferred from the $\mathrm{N}$ - to C-terminus (Fig. 2, Additional file 1). LsVe1S had an additional potential transmembrane domain and non-cytoplasmic region. Similarly, there were three hits $(\mathrm{e}=0.0)$ on contig Lsat_LaBrillante_v1_g_2266 for La Brillante. The hits corresponded to three gene models that differed in sequence from the three $V e$ genes in cultivar Salinas. Phylogenetic analyses showed that two of the ORFs grouped with maximum support with the Ve1 and Ve3 alleles in Salinas (Fig. 3) and were therefore named $L s V e 1 L$ and $L s V e 3 L$, respectively. The third gene sequence was sufficiently different from all three genes in cultivar Salinas and was therefore named LsVe4L (Fig. 3). $L s V e 1 L$, LsVe3L, and LsVe4L encode proteins measuring 1136, 503, and 1043 aa, respectively. The domains encoded by $L s V e 1 L$ and $L s V e 4 L$ were the same as for LsVe1S and LsVe3S, respectively; however, while the sequence of $L s V e 3 L$ is similar to LsVe3S, premature stop codons result in a truncated protein encoded by $L s V e 3 L$ (Fig. 2, Additional file 1).

The La Brillante and Salinas Ve alleles were then used as queries to identify homologs in diverse germplasm of cultivated lettuce. A total of $180 \mathrm{Ve}$ sequences were extracted from genome assemblies of 60 lettuce cultivars (Additional file 2). The sequences represented 21 different alleles that were identical or similar to the $V e$ alleles from La Brillante and Salinas (Fig. 4). The LsVe1L clade contained a single allele and the remaining clades contained between two and six alleles (Fig. 4, Additional file 2 ). This analysis likely underestimated the total number of $\mathrm{Ve}$ alleles because only 47 of the $186 \mathrm{Ve}$ sequences included in this study represented complete genes (Additional file 2). All cultivars contained three $\mathrm{Ve}$ genes, except cultivar Anuenue (susceptible), in which only two alleles were detected that clustered in the LsVe2 and $\mathrm{LsVe} 3$ clades, and cultivar Cobham Green (susceptible) that contained four $V e$ genes that clustered in the $L s V s 1 S, L s V e 2 S, L s V e 3 L$, and LsVe4L clades. For the remaining $L s V e$ genotypes, see Table 2.

There were substantial differences in frequencies of $L s V e$ alleles among lettuce horticultural types. For example, all tested Iceberg cultivars had the identical combination of three alleles, LsVe1S, LsVe2S, and LsVe3S, while none of the genotyped Latin accessions contained any of these alleles (Table 3) . Only six combinations of $L s V e$ alleles were detected in 62 accessions with sequenced genomes. The five combinations were found in susceptible accessions: 40 accessions with LsVe1S, LsVe2S, and LsVe3S; four accessions with LsVe4L, $L s V e 1 S$, and LsVe2S; one accession with LsVe3L, LsVe4L, and LsVe2S; one accession with LsVe3L, LsVe4L, LsVe1S, and LsVe2S; and one accession with LsVe2S and LsVe3S. In addition, one combination of alleles was found in all (15) resistant accessions LsVe1L, LsVe3L, and LsVe4L (Table 3).

Phylogenetic analyses of $\mathrm{Ve}$-encoded amino acid sequences from cultivars La Brillante and Salinas with tomato $\mathrm{Vel}$ and homologs from other Asteraceae, Cannabaceae, Malvaceae, and Solanaceae species showed that these lettuce $V e$ alleles were monophyletic with $99 \%$ bootstrap support. Two equally parsimonious trees were obtained and the tree length was 3492 steps (Additional file 3).

\section{PCR- based screening for LsVe1L and LsVe4L in 90 additional accessions}

In order to determine the prevalence of candidate resistance alleles, 90 additional accessions were screened for the presence of $L s V e 1 L$ and $L s V e 4 L$ using allele-specific PCR (Additional file 4 and Additional file 5). LsVelL-specific products were detected in six accessions and LsVe4L-specific products in 12 accessions. All accessions with LsVe1L also had LsVe4L (Table 2). LsVe3L screening was not performed because of the premature stop codons as mentioned above (Fig. 2).

\section{Diagnostic PCR assays for race 1 resistance based on LsVe1L}

LsVe $1 L$ and $L s V e 1 S$ only share $89.5 \%$ sequence similarity differing by 358 single nucleotide polymorphisms (SNPs) and two indels. The overall ratio of non-synonymous $(d \mathrm{~N}=0.0754)$ to synonymous $(d \mathrm{~S}=0.2324)$ substitutions between the two alleles was 0.3246 , providing no evidence for diversifying selection. These SNPs and indels provide multiple possibilities for allele-specific PCRbased assays. A PCR assay that selectively amplified LsVe1L was developed and validated as a marker for resistance to race 1 using selected accessions of lettuce with known $V e$ genotypes and resistance phenotypes. All PCR results were consistent with phenotypic observations and genome sequence data (Fig. 5). The LsVe1L

\begin{tabular}{|c|c|c|c|c|c|c|c|c|c|c|c|c|}
\hline 1 & $2,0,00$ & $4,0,00$ & $6,0,00$ & 8,000 & 10,000 & 12,000 & 14,000 & 16,000 & 18,000 & 20,000 & 22,000 & 24,000 \\
\hline 1 & 10,000 & 20,000 & 30,000 & 40,000 & 50,000 & 60,000 & 70,000 & 80,000 & 90,000 & 100,000 & 110,000 & 120,000 \\
\hline
\end{tabular}

Fig. 1 Partial scaffolds of lettuce cultivars La Brillante (Lsat_LaBrillante_v1_g_2266) and Salinas (Ig_9) containing LsVe genes 


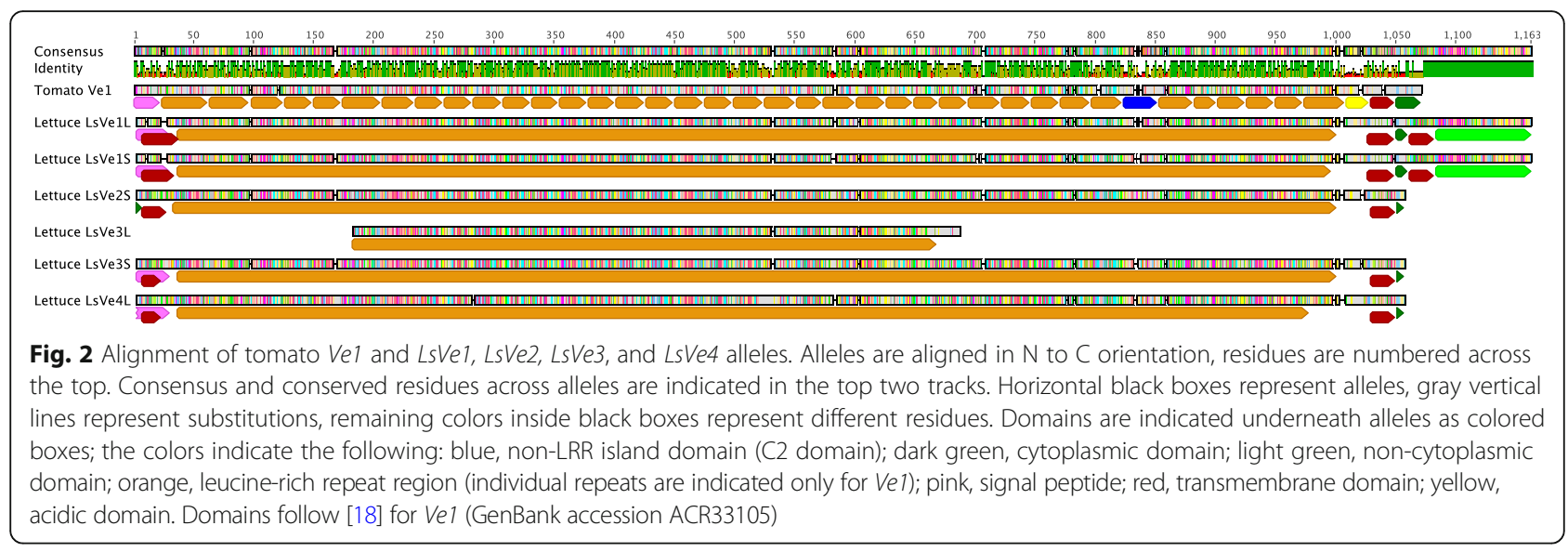

allele was detected in 21 of the 152 tested accessions and all 21 were resistant to $V$. dahliae race 1 in field experiments (Table 2). Wilt symptoms were not observed on any of the 21 accessions with the exception of cultivar Plymouth, where two out of 30 plants showed root discoloration. However, the pathogen isolated from tap root tissue of cultivar Plymouth lacked the $V$. dahliae race 1 determinant Ave1 [8], thus revealing that the symptoms were not caused by $V$. dahliae race 1 (Additional file 6). LsVe $4 L$ was present in all resistant but also some susceptible accessions (Table 2). This is consistent with $L s V e 1 L$ rather than $L s V e 4 L$ conferring resistance to $V$. dahliae race 1.

\section{Discussion}

We tested 149 accessions of cultivated lettuce and a single accession of L. serriola in field experiments. Horticultural types with the greatest number of tested accessions were iceberg (46) and romaine (36) because they are the predominant types grown in the U.S. [1]. Despite the largest number of tested accessions, none of the iceberg cultivars were resistant to Verticillium wilt. This observation complements results from a previous study that tested accessions from multiple horticultural types for resistance to $V$. dahliae race 1 [19]. Therefore, development of modern iceberg-type cultivars with resistance to $V$. dahliae race 1 is one of the top priorities for public and private breeding efforts. USDA-ARS in Salinas released iceberg breeding

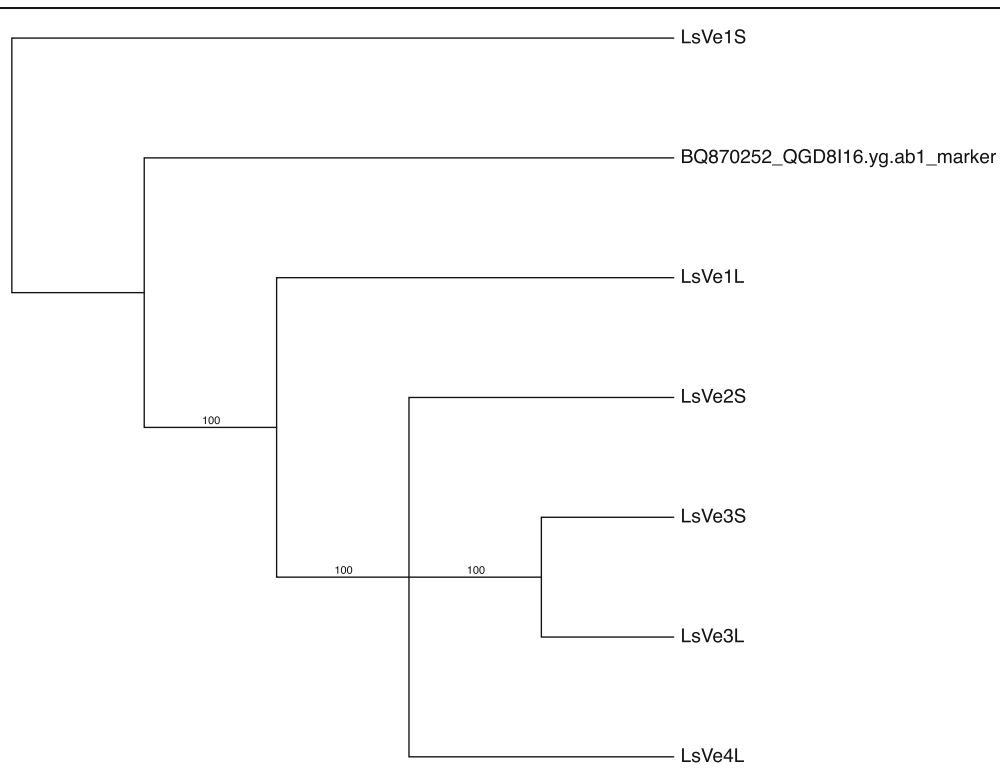

Fig. 3 Unrooted parsimony bootstrap tree of cultivars La Brillante and Salinas Ve alleles. The topology shows that LsVe1 alleles plus BQ870252_QGD8116.yg.ab1 marker (GenBank accession BQ870252) [16] group together with maximum statistical support; the LsVe3 alleles group together but LSVe4L and LsVe2S alleles do not. Bootstrap supports above $70 \%$ are indicated by the branches 


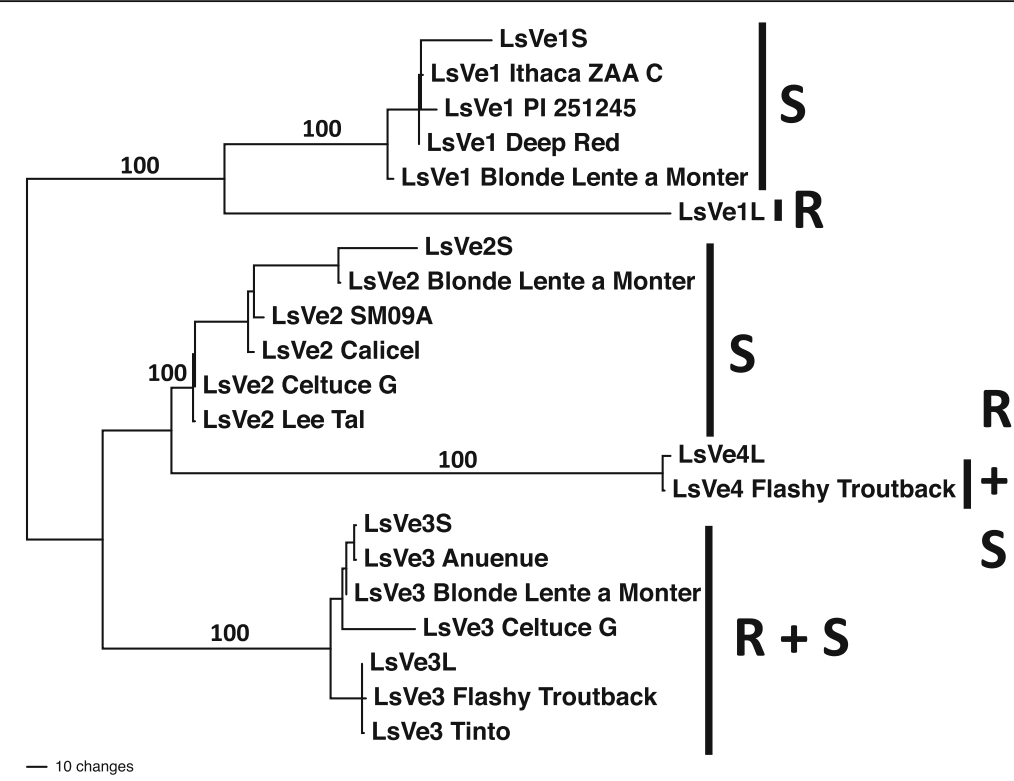

Fig. 4 Phylogenetic tree of all LsVe alleles found in this study. Only one representative of each allele is included in the tree. Shown is one of 322 most parsimonious trees measuring 1120 steps; the tree is midpoint rooted. Taxon names consist of gene name followed by accession, except for cultivars La Brillante (L) and Salinas (S), where allele names are given. Numbers by the branches are bootstrap supports above $70 \%$. Branch lengths are proportional to the number of changes occurring along the branches, the scale is given at the bottom. Association of alleles with resistance or susceptibility is indicated on the right side of vertical bars with R and S, respectively. All alleles are shown in Additional file 2

lines [20-22] with their resistance derived from cultivar La Brillante. Cultivar La Brillante is a Batavia type lettuce with a small, round head that is less dense than those of modern iceberg cultivars. Because of the certain phenotypic similarities in the shape of heads, fewer backcrosses are usually needed to develop true to type iceberg cultivars when introgressing desirable genes from Batavia accessions than would be needed if those genes were introgressed from non-heading types of lettuces. Our current analyses showed that besides cultivar La Brillante, another Batavia cultivar (cultivar Laura) can also be used for a relatively rapid development of iceberg cultivars with resistance to $V$. dahliae race 1 . Both of these cultivars contain the same combination of LsVe alleles (LsVe1L, LsVe3L, and LsVe4L).

Only two out of 36 romaine accessions were resistant to the disease in field experiments. One of the resistant accessions, cultivar Annapolis, is a dark red lettuce with a relatively small and light head that is usually grown for baby leaf production and is therefore harvested at early stages of development. The other resistant cultivar was Defender, which is green. Origin of resistance in this cultivar is unknown because it was developed through open pollination [23]. A high frequency (87.5\%) of resistance to the disease was found in Latin type accessions

Table 3 LsVe allele frequencies in lettuce horticultural types included in this study. The number of accessions examined for the presence of the particular allele is given in parentheses

\begin{tabular}{|c|c|c|c|c|c|c|}
\hline Type & LsVe1L & LsVe3L & LsVe4L & LsVeis & LsVe2S & LsVe3S \\
\hline Batavia & $0.43(7)$ & $0.50(4)$ & $0.43(7)$ & $0.25(4)$ & $0.50(4)$ & $0.50(4)$ \\
\hline Butterhead & $0.21(14)$ & $0.43(7)$ & $0.79(14)$ & $0.71(7)$ & $0.71(7)$ & $0.14(7)$ \\
\hline Green leaf & $0.11(18)$ & $0.25(8)$ & $0.11(18)$ & $0.75(8)$ & $0.75(8)$ & $0.75(8)$ \\
\hline Iceberg & $0(47)$ & $0(16)$ & $0(47)$ & $1.00(16)$ & $1.00(16)$ & $1.00(16)$ \\
\hline Latin & $0.86(7)$ & $1.00(3)$ & $0.86(7)$ & $0(3)$ & $0(3)$ & $0(3)$ \\
\hline Oil & $0(4)$ & $0(2)$ & $0(4)$ & $1.00(2)$ & $1.00(2)$ & $1.00(2)$ \\
\hline Red leaf & $0.27(15)$ & $0.43(7)$ & $0.33(15)$ & $0.57(7)$ & $0.57(7)$ & $0.57(7)$ \\
\hline Romaine & $0.06(36)$ & $0.23(13)$ & $0.11(36)$ & 0.77 (13) & $0.85(13)$ & $0.69(13)$ \\
\hline Stem & $0.33(3)$ & $0.50(2)$ & $0.33(3)$ & $0.50(2)$ & $0.50(2)$ & $0.50(2)$ \\
\hline Lactuca serriola & $0(1)$ & $N D^{a}$ & $1.00(1)$ & ND & ND & ND \\
\hline
\end{tabular}

${ }^{a}$ Not determined 


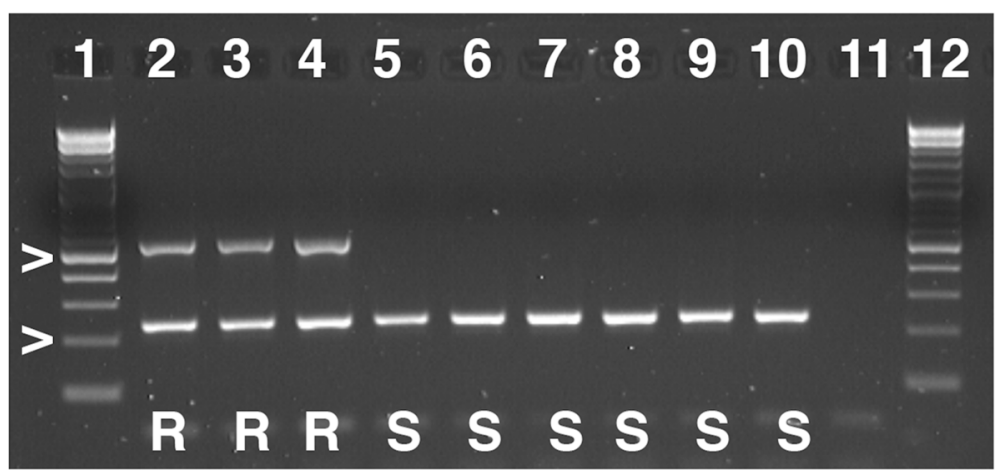

Fig. 5 LsVe1L specific PCR assay is allele-specific. Shown are results of LsVelL-specific PCR assays with selected lettuce accessions with known LsVe genotypes and resistance phenotypes. Resistance (R) and susceptibility (S) is indicated by capital letters for each accession. In all cases, the outcomes of the PCR assays were as expected from genome sequencing. Amplicon sizes are indicated by $>$ and correspond to 200 and $500 \mathrm{bp}$. Lane numbers are: 1. 2-log ladder, 2. cultivar Balady Banha (Ve genotype: LsVe1L, LsVe3L, LsVe4L), 3. cultivar Lolla Rossa (LSVe1L, LSVe3L, LSVe4L), 4. cultivar Plymouth (LsVe1L, LsVe3L, LSVe4L), 5. cultivar Cobham Green (LsVe3L, LSVe4L, LSVe1S, LSVe2S), 6. cultivar Lee Tal (LsVe4L, LsVe1S, LsVe2S), 7. cultivar Margarita (LsVe4L, LSVe1S, LSVe2S), 8. cultivar Anuenue (LsVe2S, LsVe3S), 9. cultivar Blonde Lente a Monter (LsVe1S, LsVe2S, LsVe3S), 10. cultivar Primus (LsVe1S, LsVe2S, LsVe3S), 11. negative control, and 12. 2-log ladder. PCR conditions are described in Table 4

that phenotypically resemble a small romaine lettuce with more pliable and oily leaves. Because of the phenotypic similarity between romaine and Latin types, Latin-type accessions may also be used for a relatively rapid development of romaine cultivars with resistance to $V$. dahliae race 1 . Both romaine cultivars and three sequenced Latin cultivars (Eruption, Pavane, and Little Gem) that are resistant to the disease contain an identical combination of LsVe alleles (LsVe1L, LsVe3L, and LsVe4L).

Substantially different frequencies of $L s V e 1 L$ alleles (Table 3) and resistant phenotypes (Table 1) in different horticultural types of lettuce are not unexpected considering that comparable differences were previously described for other monogenically inherited traits, such as resistance to lettuce dieback [24] and sensitivity to triforine [25]. Differences in the frequency of specific alleles among horticultural types are likely caused by the breeding approach that is used to develop lettuce cultivars. Only a few elite progenitors or founder lettuce cultivars have given rise to most of the modern commercial cultivars [26]. Each of these progenitors is frequently found in pedigrees of cultivars of the same horticultural type. Additionally, new cultivars are mainly developed by recurrent breeding within small pools of closely related germplasm of the same type [27]. Therefore, alleles present in an original progenitor(s) of a certain type are found in high frequency in cultivars of the same type, but may be absent or present in low frequency in cultivars of other horticultural types.

Our data are consistent with the LsVe1 gene identified in the cultivar La Brillante being involved in resistance to $V$. dahliae race 1 in lettuce. Among the 152 accessions included in this study, 21 were resistant to $V$. dahliae race 1 and all 21 contained the LsVe1L allele; this allele was not present in any of the susceptible accessions. The other La Brillante Ve alleles, LsVe3L and $L s V e 4 L$, were also present in all the resistant accessions, but they also occurred in two and twelve susceptible accessions, respectively. Therefore, $L s V e 1 L$ is the strongest candidate as being required for resistance to $V$. dahliae race 1 in lettuce, although our data do not exclude $L s V e 3 L$ or $L s V e 4 L$ from also being involved similarly as in tomato [12]. Complementation and knock-out studies are still required to determine the functional basis of $L s V e$-mediated resistance to $V$. dahliae race 1 .

The function and the significance of the differences between the LsVe1L and LsVe1S alleles (Additional file 7) remains to be investigated. The proteins encoded by LsVe1L and LsVe1S have the same domain organization, including the 37 extracellular, leucine-rich repeats separated by a short spacer region, as in previously characterized functional Ve proteins in other species $[11,18]$. However, in addition to sequence diversity in the extracellular LRR domain, LsVe1L has an additional Cterminal transmembrane domain as compared to $\mathrm{Ve} 1$ and $\mathrm{Ve} 2$ in tomato, suggesting that maybe LsVe1L crosses the membrane three times and terminates with a non-cytoplasmic domain instead of a cytoplasmic domain.

The distribution of disease incidence in susceptible accessions (from 0.07 to 1.00) and across horticultural types ( $>0.98$ in stem types, but only 0.17 in a single susceptible Latin) indicates a possible presence of a modifying factor or factors that affect disease incidence. Our data do not exclude the possibility of interactions between two or more $V e$ genes, similar to those reported in tomato [12]. A more detailed study of accessions with 
different frequencies of disease incidence and allelic compositions is needed to elucidate the basis of variation in disease incidence.

\section{Conclusions}

There is a critical lack of iceberg and romaine type cultivars with resistance to $V$. dahliae race 1 . Application of molecular markers can accelerate the lettuce breeding process while improving selection accuracy [28]. Therefore, the development of molecular marker assays for identification of desired genotypes is highly sought-after. The LsVe1Lspecific PCR assay developed in this study can be used for the selection of lettuce genotypes with resistance to $V$. dahliae race 1. Application of this assay allows identification of resistant genotypes in early stages of plant development (or at a seed-level) without time- and labor-intensive testing of plants in the field. This molecular marker is a valuable addition to the tools available to breeders when developing improved cultivars of lettuce.

\section{Methods \\ Lettuce accessions used for genome sequencing and PCR analysis}

A total of 152 lettuce accessions representing all major types of cultivated lettuce (Batavia, butterhead, iceberg, Latin, leaf, oil, romaine, and stem) were analyzed (Table $2)$. The majority of accessions (111) were from the United States Department of Agriculture, Agricultural Research Service (USDA-ARS) lettuce collections at Salinas, California; the remaining accessions were from a variety of sources (Table 2), including Salinas, the previously sequenced cultivar [16]. When an accession was obtained from more than one source, each provenance was considered separately in the analyses.

\section{Pathogenicity tests}

Experiments were conducted in a field infested with $V$. dahliae race 1 [16] located at the USDA-ARS station in Salinas, California. One hundred and fifty accessions were direct-seeded in a randomized complete block design with three replications. The original seed batches of previously sequenced cultivars Salinas [17] and La Brillante (this publication) were not available for field tests; therefore, seed batches used in field tests are shown as separate entries (Table 2). Each plot was $7 \mathrm{~m}$ long and consisted of two seed lines on $1 \mathrm{~m}$ wide beds standard for lettuce production in coastal California. Plant spacing was approximately $28 \mathrm{~cm}$ between seed lines and $30 \mathrm{~cm}$ between plants within a seed line. All field experiments were maintained using standard cultural practices for coastal California lettuce production. Plants were evaluated for disease incidence after reaching harvest maturity. Unless indicated otherwise, ten plants from each plot were uprooted and visually evaluated. Disease incidence was assessed by cutting taproots longitudinally and recording the number of plants exhibiting the yellowish-brown discoloration of root vascular tissues that is typical of Verticillium wilt. Absence of $V$. dahliae race 1 in cultivars with race 1-resistant genotype was confirmed by plating surface-sterilized symptomatic root tissue on NP-10 semi-selective agar medium [29] and PCR screening any resulting isolates with Ave1-specific primers [8]. Three additional experiments were performed in the same field to confirm phenotypic observations. These experiments comprised only a subset of accessions that were either symptomless in the first experiment or were used as susceptible checks. Disease incidence values from all four experiments were combined and used for statistical analyses with JMP 14.2 (SAS Institute, Cary, NC, USA).

\section{DNA extraction}

DNA was extracted using FastDNA SPIN kit (MP Biomedicals, Solon, OH, USA) for most lettuce accessions and with the CTAB method [30] for La Brillante accession 10G364-1. For the FastDNA SPIN kit method, up to 100 mg seeds ( 100 seeds) or freeze-dried leaf tissue was crushed in liquid nitrogen with a mortar and pestle, and DNA was extracted according to the manufacturer's instructions for plant material. DNA quality was assessed using gel electrophoresis ( $0.7 \%$ agarose gel), a NanoDrop spectrophotometer, and a Qubit Fluorometer (both Thermo Fisher Scientific, Wilmington, DE, USA) as per the manufacturers' instructions. DNA extraction for $V$. dahliae followed the same FastDNA SPIN kit protocol except that CLS-Y solution was used as suggested by the manufacturer.

\section{Genome sequencing and assembly}

For La Brillante accession 10G364-1, three genomic libraries were constructed, one with $180 \mathrm{bp}$ insert size (with in-house protocols) and two Nextera (Illumina, San Diego, CA, USA) 2 and $7 \mathrm{~kb}$ mate-pair libraries. All libraries were sequenced in an Illumina Hi-Seq 2000 for $100+100$ paired-end reads. Reads were directly imported into AllPaths-LG v49856 [31] and assembled using default parameters. Both mate-pair libraries were aligned to the AllPaths-LG assembly using BWA v0.7.4 [32] and these alignments were fed into SSpace v3.0 [33] together with the assembly to perform further scaffolding.

For the remaining accessions, DNA was sent to Novogene (Beijing, China) for library preparation (insert size $350 \mathrm{bp}$ ) and sequencing on Illumina HiSeq $4000 \mathrm{ma}-$ chines to generate $\sim 800 \mathrm{M}$ PE150 reads that provided approximately $25 \mathrm{x}$ whole genome coverage. Reads obtained from Novogene were further processed to remove low quality sequences using bbduk from the BBMap suite v33.65 [34]. This removed sequences with a quality score 
Table 4 PCR conditions and primer sequences used for LsVe $1 L$ and LsVe4L multiplex assays

\begin{tabular}{|c|c|c|c|c|}
\hline Target locus ${ }^{\mathrm{a}}$ & Forward primer ${ }^{b}$ & Reverse primer ${ }^{b}$ & Annealing temperature ${ }^{c}$ & Amplicon size $^{d}$ \\
\hline LSVeTL & 5'-CAA GGG CTC TAT GTC ATT CCT CC & 5'-GAC CCA TGG AAG CTG TTG GAT CT & $60^{\circ} \mathrm{C}$ & $569 \mathrm{bp}$ \\
\hline LsVe4L & 5'-CTT GTC CCA GAT AGA GTT GTC CAC C & $5^{\prime}$-CAG ACC CTG GAA ATC TाT GGT TTG A & $57^{\circ} \mathrm{C}$ & $505 \mathrm{bp}$ \\
\hline$H P P D^{1}$ & 5'-TCC CAA CTC CTC ACA CTC CTT AAT C & 5'-GTA CGG AAC AAA GAG GAA GAG CC & $57^{\circ} \mathrm{C}$ or $60^{\circ} \mathrm{C}$ & $244 b p$ \\
\hline
\end{tabular}

${ }^{a}$ The lettuce HPPD was targeted as a DNA quality control in both the LsVe1L and LsVe4L multiplex assays

${ }^{b}$ Each $25 \mu \mathrm{L}$ PCR reaction contained $1.25 \mu \mathrm{L}$ of each of the four primers at $10 \mu \mathrm{M}$ each to amplify HPPD plus LsVe1L or HPPD plus LsVe4L, $12.5 \mu \mathrm{L} 2 \mathrm{x}$ GoTaq Colorless Master Mix (Promega Corp., Madison, WI), and $7.5 \mu \mathrm{L}$ DNA template from a $1 \mathrm{ng} / \mu \mathrm{L}$ stock

'The PCR program consisted of an initial denaturation at $94^{\circ} \mathrm{C}$ for $2 \mathrm{~min}$, followed by 32 cycles of denaturation at $94^{\circ} \mathrm{C}$ for $10 \mathrm{~s}, 20 \mathrm{~s}$ at the assay-specific annealing temperature, extension at $72{ }^{\circ} \mathrm{C}$ for $1 \mathrm{~min}$, and a final extension at $72{ }^{\circ} \mathrm{C}$ for $7 \mathrm{~min}$. PCRs were set up on ice under sterile conditions and the thermocycler was preheated to $94^{\circ} \mathrm{C}$ before adding the reactions

${ }^{\mathrm{d} P C R}$ products $(8 \mathrm{LL}$ each) were run on a $1 \%$ agarose gel

below 20 from both ends of the read and eliminated reads that had less than $50 \mathrm{bp}$ after trimming. Genome assemblies were generated using MEGAHIT version 1.0.6 [35] or MaSuRCA version 2.3.2 [36]. MEGHIT was generally run with default settings and sometimes with metasensitive or bulk options in effect. MaSuRCA settings were insert size $=350$ and standard deviation $=50, \mathrm{GRAPH}_{-}$ KMER_SIZE $=101$, USE_LINKING_MATES $=0$, LIMIT_ JUMP_COVERAGE $=300, \quad$ CA_PARAMETERS $=\mathrm{cgw}$ ErrorRate $=0.15$ ovlMemory $=4 \mathrm{~GB}, \quad$ KMER_COUNT THRESHOLD $=1$, NUM_THREADS $=40, \quad J F \_S I Z E ~=10$, $000,000,000$, and DO_HOMOPOLYMER_TRIM $=0$. Assembly statistics were generated using the shell script stats.sh of BBMap version 37.68 [34].

\section{Ve gene identification and naming}

The expressed sequence tag marker QGD8I16.yg.ab1 at the Verticillium resistance 1 ( $V r 1)$ locus in lettuce [16] that has sequence similarity to the $V e$ genes of tomato was used to query the genome assemblies of $V$. dahliae race 1 susceptible cultivar Salinas [17] (assembly version 8 , available at https://genomevolution.org/coge/GenomeInfo.pl?gid=28333) and race 1 resistant cultivar La Brillante, using local nucleotide BLAST v. 2.6 [37]. The LsVe sequences from cultivars La Brillante and Salinas were then used to query the remaining lettuce genome assemblies using BLASTn. Sequence alignments were generated with MAFFT version 7.309 [38, 39] using default settings. Phylogenetic analyses were performed with PAUP 4.0a (build 159) [40] using the maximum parsimony criterion, the heuristic search option, and 10 random addition replicates. Bootstrap branch support was based on 1000 random addition replicates. Default settings were used otherwise. Protein domains were annotated using the InterPro website (https://www.ebi.ac.uk/ interpro/) [41]. Codon alignments were subjected to calculation of synonymous and non-synonymous substitution rates with PAL2NAL v. 14 [42].

\section{PCR assays}

La Brillante LsVe1L and LsVe4L-specific PCR assays were performed as follows. Each assay was a multiplex assay with two $L s V e L$-specific primers and a plant DNA control with two additional primers specific to the lettuce 4-hydroxyphenylpyruvate dioxygenase-encoding gene (HPPD), which has been used as the reference gene in real-time PCR assays [43]. PCR conditions and primer sequences are shown in Table 4.

\section{Additional files}

\begin{abstract}
Additional file 1: Alignment of tomato Vel and lettuce LsVe alleles. Domains are indicated; eLRR stands for extracellular leucine-rich repeat. Domain information for Vel is from [18]. (PDF 589 kb)
\end{abstract}

Additional file 2: LsVe alleles found in this study. Provided are names of contigs or scaffolds, identical representatives included in Fig. 4, and completeness of sequencing coverage. (XLSX $15 \mathrm{~kb}$ )

Additional file 3: Phylogenetic tree of cultivars La Brillante and Salinas Ve allele amino acid sequences and homologs from other plant families using maximum parsimony. One of two most parsimonious trees is shown measuring 3492 steps; the tree is midpoint rooted. Taxa names consist of species names followed by gene names. GenBank accession numbers are provided for sequences from other studies. Bootstrap supports above $60 \%$ are shown by the branches. Branch lengths are proportional to changes along the branches and the scale is provided. (PDF $16 \mathrm{~kb}$ )

Additional file 4: Results of LsVe1L and LsVe4L PCR screening of 90 lettuce accessions that were not genome sequenced. The legend to lane numbers is in Additional file 5. For each accession, the top gel shows results of the $L s V e 1 L$ screening, the bottom gel shows the results of the $L S V e 4 L$ screening. Resistant accessions are marked with an R. Amplicon sizes are indicated by $>$ and correspond to 200 and $500 \mathrm{bp}$. Size standard used is 2log ladder. PCR conditions are described in Table 4. (PDF 1577 kb)

Additional file 5: Legend to lane numbers in Additional file 4. For each lane, accession name, code, and PCR result are provided. (XLSX 12 kb)

Additional file 6: PCR gel demonstrating that Verticillium dahliae strains isolated from symptomatic cultivar Plymouth tap roots did not contain Ave1, the specificity determinant of race 1, and were thus not race 1. Amplicon size marker indicated by > corresponds to $1000 \mathrm{bp}$. Lane numbers are: 1. 2-log ladder, 2. and 3. Verticillium dahliae strain isolated from symptomatic cultivar Plymouth tap root, 4. and 5. V. dahliae race 2 control strain Ls.17, 6. and 7. V. dahliae race 1 control strain Ls.16, and 8. negative control. (PDF $4236 \mathrm{~kb}$ )

Additional file 7: Nucleotide sequences of six LsVe alleles from cultivars La Brillante (L) and Salinas (S). (DOCX 17 kb)

\section{Abbreviations}

HPPD: 4-hydroxyphenylpyruvate dioxygenase-encoding gene; LsVe1, LsVe2, LsVe3, and LsVe4: Lactuca sativa homologs of tomato Ve genes for resistance to Verticillium dahliae race 1; ORF: Open reading frame; RLP: Receptor-like proteins; SNP: Single nucleotide polymorphisms; Vr1: Verticillium resistance 1 locus 


\section{Acknowledgements}

The authors would like to thank Richard Bostock, UC Davis, for providing lab space; Alex Kozik, UC Davis, for help with analyses; Jose Orozco, Rebecca Zhao, and Sharon Benzen, USDA Salinas, for the excellent technical assistance with field experiments. Mention of trade names or commercial products in this publication is solely for the purpose of providing specific information and does not imply recommendation or endorsement by the U.S. Department of Agriculture.

\section{Authors' contributions}

$\mathrm{PI}, \mathrm{KVS}$, and IS conceived the study and obtained funding; PI carried out laboratory experiments and drafted the paper; PI, KVS, and IS carried out field experiments; PI and DL collected seeds and prepared samples for sequencing; $\mathrm{PI}, \mathrm{DL}$, and SRCW analyzed the sequenced data and generated the genome assemblies; PI and MC identified the Ve genes and developed the PCR-based assays; PI, MC, SRCW, RWM, KVS, and IS contributed to data interpretation and writing the paper. All authors read and approved the final manuscript.

\section{Funding}

External funding for this project was provided by the California Department of Food and Agriculture Specialty Crops Block Grants Program, Agreement Number SCB15048. The funding organization used internal and external reviewers to review the proposed study design, workplan, and budget before funding was provided. The funder had no role in the study design, data collection and analysis, or preparation of the manuscript.

\section{Availability of data and materials}

Data and results generated and analyzed during this study are included in this published article and its supplementary information files. Nucleotide sequences of six LsVe alleles from cultivars La Brillante and Salinas are provided in Additional file 7 . Sequence data of 61 genomes generated and analyzed during the current study are available in GenBank at https://www. ncbi.nlm.nih.gov/bioproject/PRJNA478460.

\section{Ethics approval and consent to participate}

Not applicable.

\section{Consent for publication}

All authors have approved the manuscript for publication.

\section{Competing interests}

The authors declare that they have no competing interests.

\section{Author details}

'Department of Plant Pathology, University of California, Davis, CA 95616, USA. ${ }^{2}$ Present address: Indigo Ag, Charlestown, MA 02129, USA. ${ }^{3}$ Genome Center, University of California, Davis, CA 95616, USA. ${ }^{4}$ Departments of Plant Sciences, Molecular \& Cellular Biology, Medical Microbiology \& Immunology, University of California, Davis, CA 95616, USA. ${ }^{5}$ United States Department of Agriculture, Agricultural Research Service, Crop Improvement and Protection Research Unit, Salinas, CA 93905, USA.

\section{Received: 4 March 2019 Accepted: 25 June 2019}

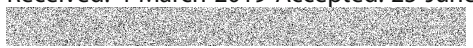

\section{References}

1. Simko I, Hayes RJ, Mou B, McCreight JD. Lettuce and spinach. In: Smith S, Diers B, Specht J, Carver B, editors. Yield gains in major US field crops. Madison: CSSA Special Publication; 2014. p. 53-86.

2. Atallah ZK, Hayes RJ, Subbarao KV. Fifteen years of Verticillium wilt of lettuce in America's salad bowl: a tale of immigration, subjugation, and abatement. Plant Dis. 2011;95(7):784-92.

3. Inderbitzin P, Subbarao KV. Verticillium wilt. In: Subbarao KV, Davis RM, Gilbertson RL, Raid RN, editors. Compendium of lettuce diseases and pests. Second ed. St. Paul: APS Press; 2017. p. 50-4.

4. Pegg GF, Brady BL. Verticillium wilts. Wallingford: CABI Publishing; 2002.

5. Inderbitzin P, Subbarao KV. Verticillium systematics and evolution: how confusion impedes Verticillium wilt management and how to resolve it. Phytopathology. 2014;104(6):564-74.
6. Vallad GE, Qin Q-M, Grube R, Hayes RJ, Subbarao KV. Characterization of race-specific interactions among isolates of Verticillium dahliae pathogenic on lettuce. Phytopathology. 2006;96(12):1380-7.

7. Gurung S, Short DPG, Atallah ZK, Subbarao KV. Clonal expansion of Verticillium dahliae in lettuce. Phytopathology. 2014;104(6):641-9.

8. de Jonge R, van Esse PH, Maruthachalam K, Bolton MD, Santhanam P, Saber MK, et al. Tomato immune receptor Ve1 recognizes effector of multiple fungal pathogens uncovered by genome and RNA sequencing. Proc Natl Acad Sci U S A. 2012;109(13):5110-5.

9. Kawchuk LM, Hachey J, Lynch DR, Kulcsar F, van Rooijen G, Waterer DR, et al. Tomato Ve disease resistance genes encode cell surface-like receptors. Proc Natl Acad Sci U S A. 2001;98(11):6511-5.

10. Wang G, Fiers M, Ellendorff U, Wang Z, de Wit PJ, Angenent GC, et al. The diverse roles of extracellular leucine-rich repeat-containing receptor-like proteins in plants. Crit Rev Plant Sci. 2010;29(5):285-99.

11. Song Y, Zhang Z, Seidl MF, Majer A, Jakse J, Javornik B, et al. Broad taxonomic characterization of Verticillium wilt resistance genes reveals an ancient origin of the tomato Ve1 immune receptor. Mol Plant Pathol. 2017; 18(2):195-209.

12. Nazar RN, Xu X, Kurosky A, Robb J. Antagonistic function of the Ve R-genes in tomato. Plant Mol Biol. 2018;98(1):67-79.

13. Chen J, Li N, Ma X, Gupta VK, Zhang D, Li T, et al. The ectopic overexpression of the cotton Vel and Ve2-homolog sequences leads to resistance response to Verticillium wilt in Arabidopsis. Front Plant Sci. 2017; 8:844.

14. Simko I, Costanzo S, Haynes KG, Christ BJ, Jones RW. Linkage disequilibrium mapping of a Verticillium dahliae resistance quantitative trait locus in tetraploid potato (Solanum tuberosum) through a candidate gene approach. Theor Appl Genet. 2004;108(2):217-24.

15. Simko I, Haynes KG, Ewing EE, Costanzo S, Christ BJ, Jones RW. Mapping genes for resistance to Verticillium albo-atrum in tetraploid and diploid potato populations using haplotype association tests and genetic linkage analysis. Mol Gen Genomics. 2004;271(5):522-31.

16. Hayes R, McHale L, Vallad G, Truco M, Michelmore R, Klosterman S, et al. The inheritance of resistance to Verticillium wilt caused by race 1 isolates of Verticillium dahliae in the lettuce cultivar La Brillante. TAG Theor Appl Genet. 2011;123(4):509-17.

17. Reyes-Chin-Wo S, Wang Z, Yang X, Kozik A, Arikit S, Song C, et al. Genome assembly with in vitro proximity ligation data and whole-genome triplication in lettuce. Nat Commun. 2017:8:14953.

18. Zhang Z, Song Y, Liu C-M, Thomma BPHJ. Mutational analysis of the Ve1 immune receptor that mediates Verticillium resistance in tomato. PLoS One. 2014;9(6):e99511.

19. Hayes RJ, Vallad GE, Qin Q-M, Grube RC, Subbarao KV. Variation for resistance to Verticillium wilt in lettuce (Lactuca sativa L.). Plant Dis. 2007:91(4):439-45.

20. Hayes RJ, Sandoya G, Mou B, Simko I, Subbarao KV. Release of three iceberg lettuce populations with combined resistance to two soilborne diseases. HortScience. 2018;53(2):247-50.

21. Hayes RJ, Maruthachalam K, Vallad GE, Klosterman SJ, Simko I, Luo Y, et al. Iceberg lettuce breeding lines with resistance to Verticillium wilt caused by race 1 isolates of Verticillium dahliae. HortScience. 2011;46(3):501-4.

22. Simko I, Hayes RJ, Bull CT, Mou B, Luo Y, Trent MA, et al. Characterization and performance of 16 new inbred lines of lettuce. HortScience. 2014;49(5):679-87.

23. Wehner TC. Vegetable cultivar descriptions for North America list 262002. HortScience. 2002;37(1):15-78.

24. Simko I, Pechenick DA, McHale LK, Truco MJ, Ochoa OE, Michelmore RW, et al. Association mapping and marker-assisted selection of the lettuce dieback resistance gene Tvr1. BMC Plant Biol. 2009;9(1):135.

25. Simko I, Hayes RJ, Truco MJ, Michelmore RW. Mapping a dominant negative mutation for triforine sensitivity in lettuce and its use as a selectable marker for detecting hybrids. Euphytica. 2011;182(2):157-66.

26. Mikel MA. Genealogy of contemporary north American lettuce. HortScience. 2007:42(3):489-93.

27. Mikel MA. Genetic composition of contemporary proprietary US lettuce (Lactuca sativa L.) cultivars. Genet Resour Crop Evol. 2013;60(1):89-96.

28. Simko I. Marker-assisted selection for disease resistance in lettuce. In: Varshney RK, Tuberosa R, editors. Translational genomics for crop breeding, Volume I: Biotic stress. Hoboken: Wiley-Blackwell Publishers; 2013. p. 267-89.

29. Kabir Z, Bhat RG, Subbarao KV. Comparison of media for recovery of Verticillium dahliae from soil. Plant Dis. 2004;88(1):49-55. 
30. Doyle J, Doyle JL. Genomic plant DNA preparation from fresh tissue-CTAB method. Phytochem Bull. 1987;19(11):11-5.

31. Butler J, MacCallum I, Kleber M, Shlyakhter IA, Belmonte MK, Lander ES, et al. ALLPATHS: de novo assembly of whole-genome shotgun microreads. Genome Res. 2008;18(5):810-20

32. Li H, Durbin R. Fast and accurate long-read alignment with burrowswheeler transform. Bioinformatics. 2010;26(5):589-95.

33. Boetzer M, Henkel CV, Jansen HJ, Butler D, Pirovano W. Scaffolding preassembled contigs using SSPACE. Bioinformatics. 2010;27(4):578-9.

34. Bushnell B: BBMap. Retrieved from sourceforge.net/projects/bbmap/. 2016.

35. Li D, Liu C-M, Luo R, Sadakane K, Lam T-W. MEGAHIT: an ultra-fast singlenode solution for large and complex metagenomics assembly via succinct de Bruijn graph. Bioinformatics. 2015;31(10):1674-6.

36. Zimin AV, Marçais G, Puiu D, Roberts M, Salzberg SL, Yorke JA. The MaSuRCA genome assembler. Bioinformatics. 2013:29(21):2669-77.

37. Altschul SF, Gish W, Miller W, Myers EW, Lipman DJ. Basic local alignment search tool. J Mol Biol. 1990;215(3):403-10.

38. Katoh K, Misawa K, Ki K, Miyata T. MAFFT: a novel method for rapid multiple sequence alignment based on fast Fourier transform. Nucleic Acids Res. 2002;30(14):3059-66

39. Katoh K, Standley DM. MAFFT multiple sequence alignment software version 7: improvements in performance and usability. Mol Biol Evol. 2013; 30(4):772-80.

40. Swofford DL. PAUP*. Phylogenetic analysis using parsimony (*and other methods). Version 4. Sunderland: Sinauer Associates; 2002.

41. Finn RD, Attwood TK, Babbitt PC, Bateman A, Bork P, Bridge AJ, et al. InterPro in 2017-beyond protein family and domain annotations. Nucleic Acids Res. 2017:45(D1):D190-9.

42. Suyama M, Torrents D, Bork P. PAL2NAL: robust conversion of protein sequence alignments into the corresponding codon alignments. Nucleic Acids Res. 2006;34:W609-12.

43. Borowski JM, Galli V, da Silva Messias R, Perin EC, Buss JH, e Silva SDA, et al. Selection of candidate reference genes for real-time PCR studies in lettuce under abiotic stresses. Planta. 2014;239(6):1187-200.

\section{Publisher's Note}

Springer Nature remains neutral with regard to jurisdictional claims in published maps and institutional affiliations.

Ready to submit your research? Choose BMC and benefit from:

- fast, convenient online submission

- thorough peer review by experienced researchers in your field

- rapid publication on acceptance

- support for research data, including large and complex data types

- gold Open Access which fosters wider collaboration and increased citations

- maximum visibility for your research: over $100 \mathrm{M}$ website views per year

At $\mathrm{BMC}$, research is always in progress.

Learn more biomedcentral.com/submissions 